\title{
PROTON INDUCTION OF GENE MUTATIONS
}

\author{
Natalia Koltovaya*, Nadya Zhuchkina, Natalia Shvaneva
}

\author{
Joint Institute for Nuclear Research, Dubna, Russia
}

\begin{abstract}
We investigated the effect of low-LET proton irradiation on gene mutations. DNA lesions were caused by the acute exposure of the cells to $150 \mathrm{MeV}$ proton irradiation (linear energy transfer of $0.539 \mathrm{keV} / \mu \mathrm{m}$ ) and ${ }^{60} \mathrm{Co} \gamma$-rays at doses up to $25 \mathrm{~Gy}$. To detect gene mutations, we used a forward mutation rate assay that detects the mutations inactivating the arginine permease gene (Can ${ }^{R}$ mutations) and a reversion assay detecting the frameshift mutations that reverts a 4-base insertion in the LYS2 gene (lys2-Bgl). The dose responses can be described by a linear relationship for both types of gene mutations. Different radiation sources that induce mutations have the relative biological effectiveness values of 1.25 for frameshift mutations and 1.69 for forward gene mutations Can ${ }^{R}$. So, the damage induced by proton irradiation of cells appears to be more serious than the damage induced by $\gamma$-irradiation, which is indicated by an increase in the yield of not only DSBs but also gene mutations.
\end{abstract}

Key words: Proton irradiation, $\gamma$ irradiation, gene mutation, frameshift, base pair substitution, yeast

DOI: $10.21175 /$ RadProc.2016.02

Space radiation is hazardous to long-duration space crews because it may cause cancer and damage DNA. The problem of protection from chronic exposure to cosmic radiation, which is primarily composed of protons (95\%), in future manned missions to Mars has not yet been solved. To model the effect of cosmic radiation on living cells, we used a proton accelerator beam at JINR (Dubna) and the eukaryotic unicellular yeast Saccharomyces cerevisiae. Based on the conservation of the fundamental molecular processes, including DNA repair, in the eukaryotic cells from yeast to humans, the data gained from studying model systems, such as yeast, can be estimate to studies of higher eukaryotic systems.

A comparison of different ionizing radiations showed that charged particles produced a greater number of DSBs compared to abasic and oxidized base clusters than ionizing photons, with protons generating the highest ratio of DSBs to abasic and oxidized damage [1]. DNA strand breaks induced by protons were predominantly repaired by homologous recombination and postreplication repair pathways [2]. There are two branches of postreplication repair: template switching (error-free) and translesion synthesis (error-prone). In this study, we describe the results of our investigations of the effect of low-LET proton irradiation on gene mutations.

The source of protons was the Phasotron at JINR (Dubna). The dose rate was $0.55 \mathrm{~Gy} / \mathrm{min}$ at the energy of $171 \mathrm{MeV}$ (150 MeV at the target with the LET of $0.539 \mathrm{KeV} / \mu \mathrm{m})$. The source of $\gamma$-rays was ${ }^{60} \mathrm{Co}$ (the dose rate of $0.7 \mathrm{~Gy} / \mathrm{min}$, the LET of $0.25 \mathrm{KeV} / \mu \mathrm{m}$ ) at the therapeutic equipment "Rokus" (JINR, Dubna). Yeast cells were exposed to protons and $\gamma$-rays in Eppendorf tubes. It was not necessary to grow cells in the dark following the exposure to protons and $\gamma$-rays as photoreactivation does not repair strand breaks. Cells were kept in ice to prevent DSB repair.

To study the kinetics of proton-induced gene mutations, several genetic yeast assays were used.
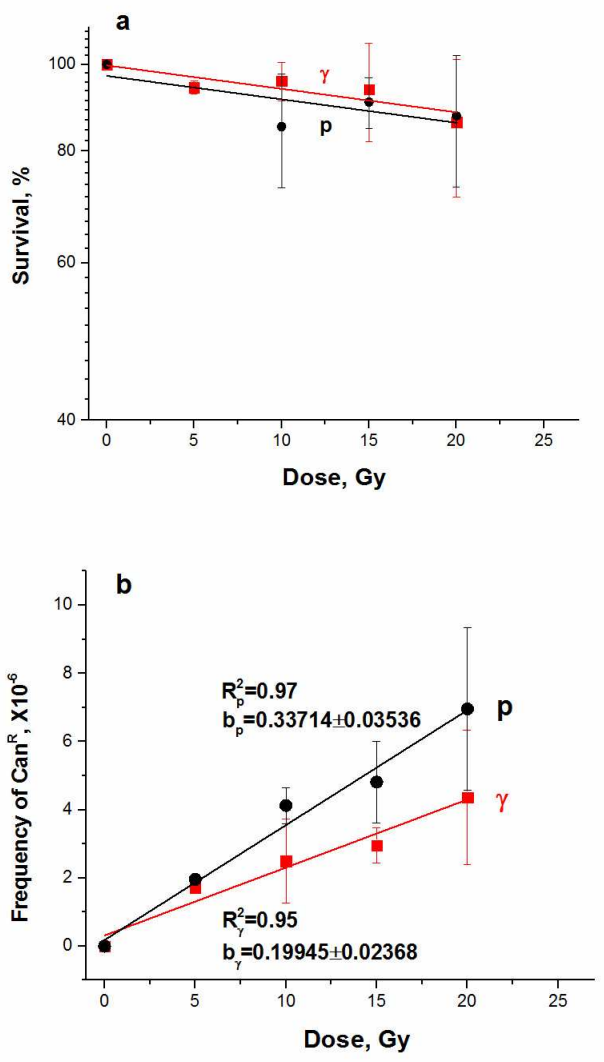

Figure 1. Survival (a) and the frequency of $\mathrm{Can}^{\mathrm{R}}$ mutants (b) were determined in the haploid yeast strain 1663 exposed to proton and $\gamma$-rays

\footnotetext{
* koltovaya@jinr.ru
} 
The forward mutation frequency at the CAN1 locus was measured to assay a very broad range of mutation types. The level of frameshift mutagenesis was assessed using the lys2 $\Delta B g l$ allele, which specifically detects net -1 frameshift mutations [3].

Mutant strains were grown nonselectively in YEPD. Selective growth was on synthetic complete medium containing $2 \%$ glucose (SM) [4] and lacking the appropriate nutrient [5]. Canavanine-resistant mutants in the forward mutation assay were identified on SM-Arg plates supplemented with $60 \mu \mathrm{g} / \mathrm{ml}$ canavanine. All growth was at $28^{\circ} \mathrm{C}$. Overnight cultures $\left(\sim 2 \times 10^{8}\right.$ cells $\left./ \mathrm{ml}\right)$ were grown in $5 \mathrm{ml}$ YEPD. Cells were resuspended in water and were kept in ice. After the exposure to proton or $\gamma$-rays, cells were plated on YEPD and appropriate SM-based selective media to assess cell survival and mutagenesis, respectively. Colonies arising on YEPD and SM plates were counted after 4-5 days of incubation, respectively. Each data point corresponds to the mean of 3 independent experiments, and error bars represent the standard deviation.

DNA lesions were caused by the acute exposure of the cells to $150 \mathrm{MeV}$ proton irradiation (liner energy transfer of $0.539 \mathrm{keV} / \mu \mathrm{m}$ ) and ${ }^{60} \mathrm{Co} \gamma$-rays at the doses up to $25 \mathrm{~Gy}$. The survival rates were exponential for both strains that were used. No differences in their sensitivity to proton and to $\gamma$-rays were determined (Fig. 1a and 2a). The survival was $\sim 85 \%$ at the dose 25 Gy.

To detect the gene mutations, we have used a forward mutation rate assay that detects the mutations inactivating the arginine permease gene (Can ${ }^{\mathrm{R}}$ mutations). Reversion assay detects the frameshift mutations that revert a 4-base insertion in the LYS2 gene. The dose responses can be described by a linear function $y=a+b x$ up to $20 \mathrm{~Gy}$ for both types of gene mutations (coefficient of determination $\mathrm{R}^{2}$ 0.95-0.98) but for frameshift mutations the curve came out to maximum at higher doses (Fig. $1 \mathrm{~b}$ and $2 b)$.

The frequency of the proton-induced $\mathrm{Can}^{\mathrm{R}}$ and frameshift mutants were elevated relative to the ones induced by $\gamma$-rays. The ratio of biological effectiveness (RBE) of proton relative to $\gamma$-ray was determined as the ratio of their curve slopes $\left(b_{p}\right.$ and $\left.b_{\gamma}\right)$. RBE for the induction of forward and frameshift mutations were $1.69 \pm 0.38$ and $1.25 \pm 0.19$ correspondently. So, the damage induced by proton irradiation of cells appears to be more serious than the damage induced by $\gamma$-ray, which is indicated by an increase in the yield of not only DSBs $[6,7]$ but also gene mutations.
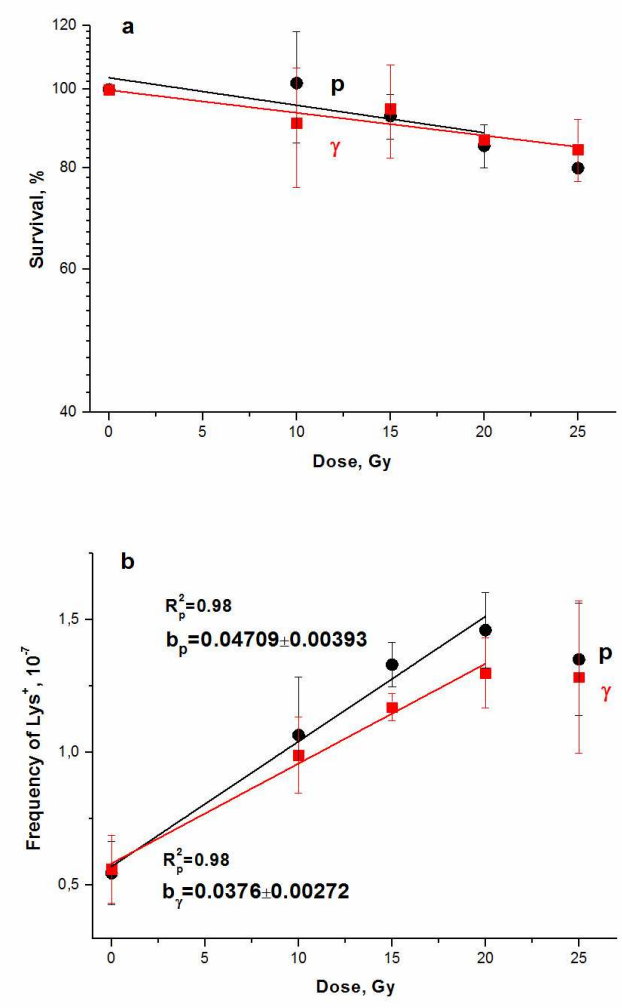

Figure 2. The survival (a) and the frequency of frameshift mutations $\mathrm{Lys}^{+}$(b) were determined in the haploid yeast strain RDKY3O23 exposed to proton and $\gamma$-rays.

\section{REFERENCES}

1. M. Hada and B.M. Sutherland, "Spectrum of Complex DNA Damages Depends on the Incident Radiation," Radiat. Res., vol. 165, no. 2, pp. 223-230, 2006

2. C. Rostek et al., "Involvement of Homologous Recombination Repair after Proton-Induced DNA Damage," Mutagenesis, vol. 23, no. 2, pp. 119-129, 2008

3. D.X. Tishkoff, N. Filosi, G.M. Gaida and R.D. Kolodner, "A Novel Mutation Avoidance Mechanism Dependent on S. Cerevisiae RAD27 is Distinct from DNA Mismatch Repair," Cell, vol. 88, no. 2, pp. 253-263, Jan. 1997

4. A.B. Devin et al., "The Start Gene CDC28 and the Genetic Stability of Yeast," Yeast, vol. 6, pp. 231-243, May 1990

5. F. Sherman, G.R. Fink and J.B. Hicks, Laboratory Course Manual for Methods in Yeast Genetics, New York (NY), USA: Cold Spring Harbor Laboratory, 1986

6. A. Antoccia, A. Sgura, M. Cavinato, R. Cherubini and C. Tanzarella, "Cell Cycle Perturbations and Cytogenetic Damage Induced by Low Energy Protons in Human Primary Fibroblasts," Rad. Prot. Dosim., vol. 99, no. 14, pp. 197-198, 2002

7. C. Di Pietro et al., "Cellular and Molecular Effects of Protons: Apoptosis Induction and Potential Implications for Cancer Therapy," Apoptosis, vol. 11, no. 1, pp. 57-66, Jan. 2006 